\title{
Gas flows within the Galaxy
}

\section{Francoise Combes}

LERMA, Observatoire de Paris, 61 Av. de l'Observatoire, F-75014, Paris

email: francoise.combes@obspm.fr

\begin{abstract}
In the recent years, more and more sophisticated models have been proposed for the gas distribution and kinematics in the Milky Way, taking into account the main bar, but also the possible nuclear bar, with the same or different pattern-speed. I review the success and problems encountered by the models, in particular in view of the new discovery of a symmetrical far-side counterpart of the $3 \mathrm{kpc}$ arm. The inner part, dominated by the bar, and the outer parts, dominated by the spiral arms, can be observed from a virtual solar position, and the errors coming from kinematical distances are evaluated. The appearance of four arms could be due to a deprojection bias.
\end{abstract}

Keywords. Galaxy, dynamics, interstellar medium, bars, kinematics

\section{Introduction}

Reconstruction of the Galaxy's spiral arms is difficult given our internal perspective. Distances of the various features or objects are derived through a kinematical model, with near-far ambiguities inside the solar circle. One of the most successful models is that from Georgelin \& Georgelin (1976) of four tightly-wound arms, traced by OB associations, optical or radio HII regions and molecular clouds. The best tracer of the Galaxy structure is the gas, atomic (HI, Liszt \& Burton 1980) and molecular (CO surveys, Dame et al. 2001), because of its low velocity dispersion, and its confinement to the plane.

Position-velocity (P-V) diagrams are particularly instructive, revealing the highvelocity $\left(\Delta V=560 \mathrm{~km} \mathrm{~s}^{-1}\right)$ Central Molecular Zone (CMZ) near zero longitude, with a molecular ring, connecting arm, $3 \mathrm{kpc}$ arm, etc. The existence of a bar has long been suspected from non-circular motions towards the center, and has been directly confirmed by COBE and 2MASS (e.g. Lopez-Corredoira et al. 2005). Near-infrared images show clearly the peanut bulge, which is thought to be formed through vertical resonance with the bar (e.g. Combes et al. 1990). The CMZ has a peculiar parallelogram shape in the $\mathrm{P}-\mathrm{V}$ diagram (Bally et al. 1988), that has been first interpreted in terms of cusped $x_{1}$ and almost circular $x_{2}$ periodic orbits, and associated gas flows, by Binney et al. (1991). Then Fux (1999) carried out fully self-consistent N-body and hydrodynamical simulations of stars and gas to form a barred spiral, and fit the Milky Way. He succeeded remarkably to reproduce the $\mathrm{HI}$ and $\mathrm{CO} \mathrm{P}-\mathrm{V}$ diagrams with a bar pattern speed of about $40 \mathrm{~km} \mathrm{~s}^{-1} \mathrm{kpc}^{-1}$, implying corotation at $5 \mathrm{kpc}$, and an ILR producing the $x_{2}$ orbit inside $1 \mathrm{kpc}$. The spiral structure has essentially 2 arms starting at the end of the bar.

\section{More recent developments}

Both external galaxies and simulations frequently show evidence for several pattern speeds in the disk and a spiral is expected to rotate slower than the bar (Sellwood \& Sparke 1988). For example, by modelling gas flow in a fixed potential Bissantz et al. (2003) conclude that in the Galaxy $\Omega_{\mathrm{p}}=60 \mathrm{~km} \mathrm{~s}^{-1} \mathrm{kpc}^{-1}$ and $20 \mathrm{~km} \mathrm{~s}^{-1} \mathrm{kpc}^{-1}$ for the bar and spiral, respectively. Amores et al (2009) notice that there is a ring gap in the 
HI distribution at about $8.3 \mathrm{kpc}$, outside the solar circle at $7.5 \mathrm{kpc}$, and propose that it corresponds to the corotation (CR) of the spiral, which will then have a pattern speed of $\sim 25 \mathrm{~km} \mathrm{~s}^{-1} \mathrm{kpc}^{-1}$. Simulations of gas in a barred spiral do not show ring gaps at CR, but depopulated regions at the corresponding Lagrangian points, which could correspond to the observations; there is no gap in the azimuthally averaged $\mathrm{HI}$ and $\mathrm{CO}$ gas surface density.

In the 2MASS star counts, a nuclear bar has been found by Alard (2001), and there is a corresponding CO nuclear bar (Sawada et al. 2004). New simulations of gas flow in a two-bar models have been done by Rodriguez-Fernandez \& Combes (2008), who find a best fit when the two bars are nearly perpendicular, and the bar-spiral pattern is about $35 \mathrm{~km} \mathrm{~s}^{-1} \mathrm{kpc}^{-1}$ (similar to Fux, 1999). The model shows the far-side twin of the $3 \mathrm{kpc}$ arm, which has just been discovered in the CO P-V diagram (Dame \& Thaddeus 2008). It reproduces also the connecting arm (characteristic leading dust lanes along the bar). No evidence is found of lopsidedness in the stellar potential, and the CO lopsidedness must be a purely gaseous phenomenon.

\section{Remaining problems}

New reconstruction of the spiral structure in the galactic plane have been attempted in the HI gas (Levine et al. 2006), and in the CO gas (Nakanishi \& Sofue 2006, Englmaier et al. 2009). The best fit could be two arms, starting at the end of the bar, with a pitch angle of $12^{\circ}$, although four arms are still possible. Pohl et al. (2008) have tried novel deprojections, by simulating the gas flow with SPH in a bar potential, and obtaining distances with a kinematic model derived from the non-circular velocity field obtained. A test of the procedure with a 2 arms + bar fiducial model, with only one pattern speed, retrieves after deprojection a four arms spiral. These recent efforts demonstrate further the difficulty of disentangling distances and dynamical effects. It is still possible that several patterns exist in the Galaxy. Other prominent features have not yet been interpreted, such as the warp or tilt of the nuclear structure, or its lopsidedness.

\section{References}

Alard, C., 2001, A\& A, 379, L44

Amores, E. B., Lépine, J., \& Mishurov, Y., 2009, MNRAS, in press (astro-ph:0907.4822)

Bally, J., Stark, A. A., Wilson, R. W., \& Henkel, C., 1988, ApJ, 324, 223

Binney, J., Gerhard, O. E., Stark, A. A., Bally, J., \& Uchida, K. I., 2001, MNRAS, 252, 210

Bissantz, N., Englmaier, P., \& Gerhard, O., 2003, MNRAS, 340, 949

Combes, F., Debbasch, F., Friedli, D., \& Pfenniger, D., 1990, A\& A, 233, 82

Dame, T., M., Hartmann, D., \& Thaddeus, P., 2001, ApJ, 547, 792

Dame, T., M. \& Thaddeus, P., 2008, ApJ, 683, L143

Englmaier, P., Pohl, M., \& Bissantz, N., 2009, Mem Societa Astron Italiana (astro-ph:0812.3491)

Fux, R., 1999, A\&A, 345, 787

Georgelin, Y. M. \& Georgelin, Y. P., 1976, $A \& A 49,57$

Levine, E. S., Blitz, L., \& Heiles, C., 2006, Science, 312, 1773

Liszt, H. \& Burton, B., 1980, ApJ, 236, 779

Lopez-Corredoira, M., Cabrera-Lavers, A., \& Gerhard, O., 2005, A\&A, 439, 107

Nakanishi, H. \& Sofue, Y., 2006, PASJ, 58, 847

Pohl, M., Englmaier, P., \& Bissantz, N., 2008, ApJ, 677, 283

Rodriguez-Fernandez, N. \& Combes, F., 2008, A\& $A$,

Sawada, T., Hasegawa, T., Handa, T. \& Cohen, R. J., 2004, MNRAS, 349, 1167

Sellwood, J. A. \& Sparke, L. S., 1988, MNRAS, 231, 25p 